\title{
Graphical Research Models in the Information Systems Discipline
}

\author{
Samuel Kießling \\ University of Innsbruck \\ samuel.kiessling@uibk.ac.at
}

\author{
Kathrin Figl \\ University of Innsbruck \\ kathrin.figl@uibk.ac.at
}

\author{
Aliaksei Miniukovich \\ University of Trento \\ aliaksei.miniukovich@unitn.it
}

\begin{abstract}
Graphical models facilitate communicating hypothesized or tested relationships between variables and are welcome in information systems publications. However, insufficient knowledge exists about design conventions for such models, lowering their communicative effectiveness. This paper investigates how graphical research models are used in the information systems literature. Theoretically, the article bears upon the perspective of prototypicality and cognitively effective design of conceptual modeling notations. Based on an analysis of 134 research models from 589 articles in information systems journals, we tentatively demonstrate prototypical features of visual research models and outline many unique graphical variations. We develop a set of hypotheses on how prototypicality influences preferences for research models and their comprehensibility and describe how we intend to test these hypotheses empirically. A broader goal of this research is to develop an effective modeling notation for research models to support researchers in constructing unambiguous visual models for their research.
\end{abstract}

\section{Introduction}

Developing a graphical research model is a typical part of the research process for many information systems researchers and such a visualization gives "an overview of the factors being studied in an empirical setting, such as the independent and dependent factors in an experiment, the important theoretical constructs and their relationships in a survey, or the set of hypotheses or propositions to be tested, explored or falsified" [1, p. 130]. In many cases, graphical research models may help readers to understand the research setting of an empirical study more efficiently without having to read the article in detail, especially because research models depict all variables in a single overview [2].
There is hardly any scientific investigation on graphical aspects of research models, despite different research fields extensively using such visual displays in their publications. Schraw and Gutierrez's [3] content analysis is a rare exception, as they investigated visual displays in the Journal of Educational Psychology between 2010 and 2014, and included research models as one type of visual display. They also pointed out that visual displays are "underresearched" given their frequent use and relevance. Insights into how research models can be used appropriately as graphical tools would help researchers to better communicate their research and help other researchers inside and outside the research discipline, as well as students and other interested readers, to read (and learn to read) such visual representations more easily [3]. Moreover, such consistent representations of research models help a researcher in a discipline to know better what we know and facilitate both the replication of research and the work on literature and meta-evaluations.

Although many IS research articles employ graphical diagrams, and conceptual modeling is a relevant IS research stream, it is surprising that almost no efforts have been undertaken to develop a common modeling notation for research models so far. A notable exception is presented by Mueller [4], who describes a meta-model for causal theories and also proposes a visual notation for describing theories which could be used to depict also research models. However, in this research no rationale was given why the specific symbols were chosen.

Language is the medium for creating a common ground [5] as it facilitates a shared understanding of concepts. The choice of a modeling notation is particularly relevant, because "the world (reality) is never given to us in and of itself, but only through interpretation in some language" [6, p. 148]. A modeling notation well defined and agreed upon by researchers would fundamentally improve research model comprehension and research idea communication within a research discipline.

Such a modeling notation should facilitate creating models that a researcher would understand 
intuitively, quickly and effortlessly. Following semantic and graphical conventions existing in the research community - i.e., making a model as typically-looking for the community as possible would be essential for the effortless model comprehension. We expect that since graphical research models have long been used in IS, the prototypical features - i.e. established visual conventions - of such models have already evolved and we can integrate them in the modeling notation. These features are a suitable basis for developing future modeling notations, as they are likely to improve model idea-communication effectiveness: recent research in web design has showed that following visual conventions [7] and increasing prototypicality [8] improves webpage user performance.

This paper develops insights into the effects of prototypicality on research models, ultimately aiming at developing a standardized modeling notation for research models in IS. Research on modeling notations has not yet explicitly addressed prototypicality, since the concepts such as familiarity and experience with a specific notation are focused on when an explicit notation already exists [9]. However, researchers have implicitly noted the relevance of prototypicality, e.g., Scaife and Rogers [10, p. 199] stated that "[a] circuit diagram, an architectural plan or a mathematical notation comprise a set of meaningless symbols to the uninitiated; they only take on their intended meaning through learning the conventions associated with them." Psychological research further showed that the mere exposure of stimuli leads to less effortful, faster perceptual processing [11], which provides a theoretical explanation why prototypical research models should be advantageous to non-prototypical models in research communication effectiveness.

If, on the other hand, one relies only on the status quo to derive a modelling standard, there is also a danger of perpetuating a suboptimal status quo, e.g. to continue randomly established modelling conventions which actually have a low cognitive effectiveness and which could be improved. We therefore also intend to identify constructive, positive deviations [see e.g. 12], i.e. features of research models that are rarely used, but beneficial from a cognitive point.

The contribution of this paper is two-fold. We first formulate hypotheses on whether prototypicality increases research model comprehensibility as well as acceptance among researchers, which we will test in our future work. To be able to investigate the effect of prototypicality in research models, we then focus our attention on identifying what constitutes prototypicality in a second step. Therefore, this paper also provides preliminary results on the identification of prototypical features of research models based on a sample of IS research models, which we will refine in future work and test empirically using an eyetracking experiment. Finally, we outline further steps on how we intend to test our hypotheses in detail.

\section{Graphical research models}

On the one hand, one objective of graphical research models is to visualize variables, which are measurable representations of abstract constructs, i.e. operationalized theoretical ideas [13]. On the other hand, they want to visualize hypotheses, which are described as "suggested linkages between constructs" [1, p. 19] or as "testable relationship between two or more variables" [1, p. 21]. In case of research models, we prefer the latter definition, because it emphasizes the measurable characteristic of these initially abstract constructs. Furthermore, research models can depict, for instance, whether constructs are uni- or multi-dimensional and which indicator variables are used to measure latent variables. Variables differ in their "nature of association with each other" [13, p. 20]. In short, independent variables (causes) have an effect on dependent variables (effects). Researchers try to hold other extraneous variables that affect the dependent variable constant or try to monitor and measure them as control variables. Moderating (or intervening) variables influence the strength of the relationship between an independent variable and a dependent variable; such an effect can be represented, for example, as interaction effect in an analysis of variance. A mediating variable can explain the relationship between an independent variable and a dependent variable.

Graphical research models represent scripts of implicit underlying domain-specific modeling languages. Compared to general-purpose graphical modeling languages such as UML (Unified Modeling Language), which can be used to model different perspectives for almost any kind of (information) system, domain-specific modeling languages are tailored for use by people in a particular domain. A domain-specific modeling language "directly represents the problem space by mapping modeling concepts to domain concepts" [14, p. 19]. It matches vocabularies and mental representations of the domain experts and can, therefore, be a powerful and easy-to-use tool in a certain domain. Generally, a graphical modeling notation offers "a set of graphical symbols (visual vocabulary), a set of compositional 
rules (visual grammar), and definitions of the meaning of each symbol (visual semantics)" [15, p. 756] to form valid expressions, i.e., diagrams. Graphical symbol include "1D graphic elements (lines), 2D graphic elements (areas), 3D graphic elements (volumes), textual elements (labels) and spatial relationships" [15, p. 757]. Hitchman [16] has noted that the development of modeling notations often neglects theoretical considerations and that authors sometimes document no reasons or design rationale for choosing particular symbols. When considering the use of research models with selfinvented graphical elements in research articles as an implicit proposal of a modeling notation, we could gain a similar impression: there is an unconscious design culture of research models in the information systems (IS) discipline, which researchers do not explicitly reflect upon. Obviously, researchers use examples of previous research models to construct their own research models. However, typically, researchers neither reference a modeling notation they used and only seldom explain the meaning of symbols and their respective relationships in their articles. Research models remain ambiguous; hence readers may find it hard to interpret them, e.g., when relationships between variables represent a hypothesized causal relationship or why variables have different shapes. Mueller [4, 17] as a rare exception discusses modeling approaches for causal theories in the IS field, e.g. for modeling theories, inter-theory relationships, theory evolution, and relationships between causal theories and empirical data. His proposal for theory visualization describes modeling conventions that remind of research models and could partly also be used to visualize research models [4, p. 4911]: "Constructs are visualized as ovals, and causal propositions are represented as arrows with a symbol and color indicating the sign [...] interaction effects are visualized by an arrow leading to the proposition [...]"'.

Other domains have successfully demonstrated how powerful the establishment of an accepted visual modeling standard can be, such as UML for the software domain or BPMN (Business Process Model and Notation) for the business process domain. Proposals of new diagram types as the entityrelationship model for database design [18] are capable of advancing a field of research. In the field of scientific research, there are visually standardized representations that have been able to establish themselves, e.g., the Prisma flow diagram with which selection criteria are to be described in systematic reviews and meta-analyses [19].

\section{Hypotheses development}

\subsection{Prototypical features of research models}

Prototypicality describes the extent to which an exemplar is perceived to represent a group or category [20]. The notion of prototypicality originated from the research on the prototype theory of concepts [21], which described concepts - entities that are used in thought and reasoning - as structured sets of properties that these concept instances were likely to possess. For example, it is quite likely that the concept instance "bird" has the set of properties "flying" and "laying eggs". In addition, prototypicality correlates with familiarity, since common examples are highly likely to be prototypical. However, prototypicality is different from familiarity because it is likely that one will remember atypical examples as well since they seem familiar [20]. Most researchers used prototypicality (or typicality) as the opposite of novelty (or newness) $[22,23]$, whereas others tried to distinguish the two as independent dimensions [24], which, however, have a strong negative impact on each other. We follow the former approach and treat prototypicality and novelty as the opposites of a single dimension.

Previous research has implied that family resemblance - the proportion of attributes shared between an item and a group - determines the prototypicality of an item [25]. Therefore, estimating family resemblance requires defining and outlining relevant attributes. A frequency value of such attributes - i.e., the chance of encountering an item as a member of a category - could be used for this, since it is considered to be a significant predictor of prototypicality [20]. This could also give an indication of which attribute is central to a category. The core of our study is to perceive category membership as graded, with some visual research models closer to the category center (i.e., the category prototype) and others further away from the center. Prototypicality in our context relates to the underlying semantic constructs presented in the research models (e.g., an independent variable) and the graphical symbols used to represent them (e.g., a rectangle) [15].

We next turn to the effect of prior exposure to examples of research models on design decisions for research model creation. Formatting guidelines, textbooks on research methods or statistical tools that require visualizing research models could potentially influence researchers to choose particular geometrical shapes for depicting research models. Rather as a side note, incidentally, the ICIS 2019 paper template is a 
case in point for employing rectangular shapes in their visual research model, which subsequently could encourage researchers to adopt this "modeling notation." The ECIS 2019 paper template, on the other hand, depicts a research model with ellipses. All templates of the IS basket journals we searched do not contain any examples of graphical research models. In this context, the research stream on "functional fixation" - a cognitive bias to use an object as it is normally used - has demonstrated how prior examples influence design tasks [26]. While we do not expect prior examples to have a negative effect, we still deem it necessary to look at possible influence factors, as there might exist different subgroups of "prototypical" research models. Jansson and Smith [27], for instance, found that designers tend to conform to examples provided to them in a conceptual design task.

In light of these results, we assume that researchers will adhere to the research models exposed to previously. In particular, we hypothesize that these prototypical visual features of research models in papers may differ slightly depending on the statistical methods used. Papers employing SEM - e.g., partial least square (PLS) analysis - typically include the concept of indicator variables, which is not used when adopting other analysis methods. The use of ellipses in research models is probably inspired by path diagrams, e.g., Remler et al. [28] prompt path diagrams to use ovals for variables and cause-and-effect arrows for representing relationships. That could be a reason for some SEM tools offering ovals for modeling (latent) variables. Based on their observations of research style guidelines, Pastor and Finney [29, p. 112] note that "researchers are not normally encouraged to include path diagrams in their publications unless the statistical model being employed is an obvious member of the SEM family (e.g., path analysis, models with latent variables)." Due to their usefulness and ability to convey complex information more easily than texts or in mathematical model equations could, Pastor and Finney [29] strongly advocate that researchers should use path diagrams more often to represent research models, even if they use statistical methods other than SEM.

Following from the discussion above, we seek to address three research questions that serve as a foundation for our paper:

RQ1: Which semantic constructs do "prototypical" research models in the IS discipline depict?

RQ2: What do "prototypical" research models look like in the IS discipline?
RQ3: Do "prototypical" research models differ for papers employing SEM from papers using other statistical methods?

Based on identifying and answering these research questions it will be possible to test the following hypotheses.

\subsection{The influence of prototypicality on preference and comprehension of research models}

The frequency of instantiation might account for such a part, as it is a significant predictor of prototypicality [20], which refers to the chance of encountering an item as a member of the category, and determines what is learned to be the central exemplar of the category.

The processing fluency theory explains the link between prototypes and user preference. The theory asserts that the effort to mentally process a stimulus determines the liking of that stimulus [30]. The stimulus prototypicality - along with other stimulus aspects, such as the cognitive complexity of the notational elements and one's experience with it determines the amount of mental processing effort of a research model. This effort, in turn, determines subconscious liking and feeling of familiarity, which translates into positive attitudes towards specific research models. We expect this preference to be reflected in further "subjective" measurements in the form of preference for it. For example, research in psychology has shown that prototypicality influences product preference [31]. Reber, Schwarz and Winkielman [11, p. 371] posit that "numerous studies confirm that prototypical and "average" forms are preferred over nonprototypical ones." In addition, we consider it as relevant in which group graphical conventions are established for a research model. As we focus on prototypicality of research models in the IS discipline, members from other research communities might not be familiar with the conventions that have evolved in the IS community; thus, for them, these models would not constitute "prototypical" research models. Also from the point of view of the scientific exchange of different research disciplines, it is relevant to know how other research communities perceive prototypical research models of the IS discipline. Hence, we hypothesize:

H1a: Information systems researchers prefer research models with higher prototypicality.

H1b: The preference for research models with higher prototypicality is higher for information systems researchers than for information systems 
students and researchers from outside of information systems.

Prototypicality may also be crucial for improving the comprehensibility of research models - the amount of mental effort to understand a model -, and a research model is useless if the reader cannot understand it. We draw on the principle of semantic transparency to explain the relationship between prototypicality and comprehension. Semantic transparency refers to how intuitively users associate the meaning of a symbol with its visual appearance [15], and runs on a continuum from semantic perversity (where users infer an opposite or different meaning from a visual symbol or a visual relationship) to semantic immediacy (where users immediately understand the meaning of a visual symbol or a visual relationship) [15]. This contributes to the ease of mental processing of the symbol (cf., the link between prototypicality and ease of processing [32], which then translates in the effort of understanding.

Most symbols used in research models are neither semantically immediate nor do they provide cues on their meaning. Using design conventions could improve comprehensibility, particularly for research diagrams and models, which describe abstract constructs that cannot be demonstrated with realistic pictures, but require learned, convention-based visual symbols [33]. Commonly encountered symbols and visual features determine a convention, and adherence to the convention determines visualization prototypicality. Higher prototypicality implies higher adherence, and thus, higher comprehensibility. Furthermore, the schema theory presumes knowledge to be organized in mind in schemata - patterns of preconceptions associated with categories and individual stimuli [34, 35]. A more prototypical stimulus is associated more strongly with the preconceptions about its category than a less prototypical stimulus.

The higher comprehensibility of prototypical research models may not be valid for all readers, especially those outside the research area in which a prototypical representation has developed. Scaife and Rogers [10, p. 210] note that the benefits of graphical representations are "due to years of practice of perceptual processing of visual stimuli and the learning of graphical conventions." Research on modeling notations has demonstrated in many studies that experience and familiarity with modeling notation are linked to model comprehension [9]. Researchers working in an area where prototypical research models have evolved encounter them regularly and have learned the meaning of different symbols used while other potential readers may not. Therefore, we posit:

H2a: Information systems researchers better understand research models with higher prototypicality.

H2b: The comprehensibility of research models with higher prototypicality is higher for information systems researchers than for information systems students and researchers from outside of information systems.

\section{Preliminary steps to identify prototypical features of research models used in the IS literature}

\subsection{Search strategy, procedure, and sample}

We solely extracted research models from the most prominent journals. Therefore, so far, we have downloaded all articles from the following journals of the years 2016-2017: European Journal of Information Systems (EJIS), Information Systems Journal (ISJ), Information System Research (ISR), Journal of Management Information Systems (JMIS), Journal of Strategic Information Systems (JSIS) and MIS Quarterly (MISQ), Journal of the Association for Information Systems (JAIS) and the Journal of Information Technology (JIT). Additionally, we screened all articles for visual research models manually. Within this data basis, we spotted 132 of 589 articles containing visual research models; thus $22 \%$ of articles included at least one research model (two articles even included two research models, totaling in 134 research models).

The first step was to categorize constructs, all visual elements and variables applied in the research models which was done by three coders. We detailed the visual characteristics of variables, perceptual grouping of variables (in the form of a common region [36]), relationships between variables, occurrences of labels for hypotheses and reading direction. As pointed and rounded edges were used interchangeably, we combined them in our analysis. We characterized the graphical visualization employed for the different types of variables (e.g., ellipses, squares or another form) as well as for the different relationships between them (e.g., edges with arrowheads or lines) in detail. The frequency of each graphical visualization can then help us to determine a favored "prototypical" research model in the IS discipline. 


\subsection{Preliminary analysis and discussion}

Figure 1 presents a research model with high prototypical features and Figure 2 a model with low prototypical gradation according to our preliminary analysis as described below.

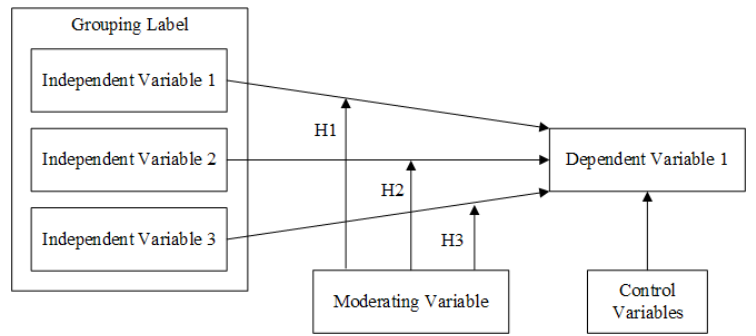

Figure 1 Derived example of a model with high prototypicality

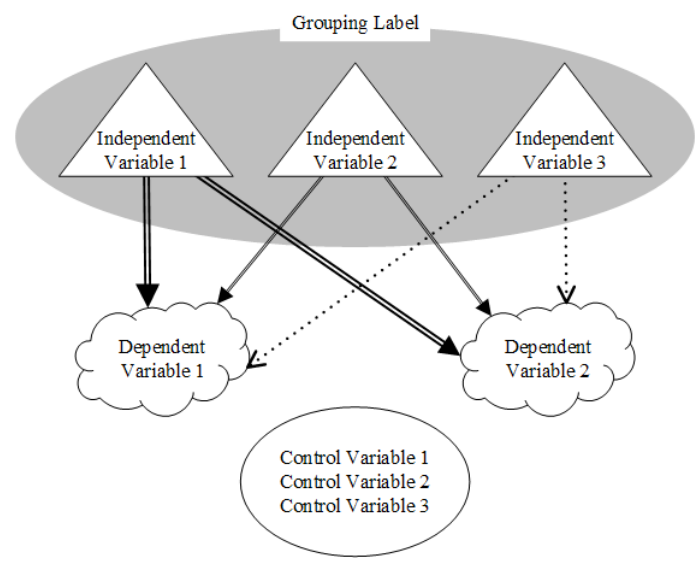

Figure 2. Derived example of a model with low prototypicality

By definition, prototypicality is the distance between a research model and a category prototype within a feature space. Estimating such distance requires defining (1) a feature space, (2) a distance measure, and (3) a category prototype. First, we established the feature space by analyzing the distinct features of the research models at hand. Table 1 differentiates between the following feature spaces: variables, grouping of variables, relationship between variables, occurrence of labels for hypotheses, and reading direction. We also identified sub-feature spaces, for instance, symbol choice or shape demarcation. The number of occurrences for a specific manifestation of a feature was selected as the distance measure. Looking at the number, the category prototype is defined by the highest number of occurrences. However, based on the number of manifestations we also defined two additional gradations for prototypicality: medium and low. Each research model needs to be checked against the predetermined gradations for prototypicality.

A lower prototypicality of a certain feature leads to a greater variation of the possible feature characteristic. When looking at shape choices with low prototypicality, for instance, researchers use various abstract symbols like pyramids or clouds. High prototypical features, on the other hand, enjoy a certain constancy in their number of different characteristics. Rectangles, e.g., are represented with pointed and rounded edges. In comparison to a completely different shape, this represents a much smaller nuance, which is why we consolidated them. Since our overall goal is to propose a unified model notation for the IS discipline, the results of the table can be used to create high and low prototypical models to highlight their idiosyncrasies.

High prototypical features are also in line with the symbols proposed by Mueller [4] to visualize theories: rectangles with rounded edges (labelled ovals in [4]) for constructs and directed edges (labelled arrows in [4]) for relationships. In contrast to his proposal, we note that research models in our dataset include not only "constructs", but typically several types of variables (e.g. control, independent and dependent variables). Concerning grouping of variables, Mueller [4, p. 4911] has mentioned that "hypernymic propositions are visualized as enclosures of the subconstruct by the superconstruct", and has used a rectangle with rounded edges and a solid line to represent it, which is also the most common choice for research models.

Table 1. Preliminary extraction of prototypical features of research models (based on a sample of 134 visual research models).

\begin{tabular}{|c|c|c|c|}
\hline \multicolumn{4}{|c|}{ Variables (Constructs) } \\
\hline Prototypicality & High & Medium & Low \\
\hline $\begin{array}{r}\text { Symbol Choice } \\
\text { (Independent, } \\
\text { Dependent, } \\
\text { Moderating, } \\
\text { Mediating) }\end{array}$ & $\begin{array}{l}\text { Rectangle } \\
(69 \%)\end{array}$ & $\begin{array}{l}\text { Ellipse } \\
(27 \%)\end{array}$ & $\begin{array}{l}\text { No symbol, } \\
\text { stand-alone } \\
\text { symbol } \\
(4 \%), \\
\text { graph }\end{array}$ \\
\hline $\begin{array}{r}\text { Symbol } \\
\text { Differentiation }\end{array}$ & $\begin{array}{l}\text { One } \\
\text { symbol } \\
(59 \%)\end{array}$ & $\begin{array}{l}\text { Two } \\
\text { symbols } \\
(35 \%)\end{array}$ & $\begin{array}{l}\text { More than } \\
\text { two } \\
\text { symbols } \\
(6 \%)\end{array}$ \\
\hline $\begin{array}{c}\text { Control } \\
\text { Variable }\end{array}$ & $\begin{array}{l}\text { Rectangle } \\
(82 \%)\end{array}$ & $\begin{array}{l}\text { Without } \\
\text { symbol } \\
(11 \%)\end{array}$ & $\begin{array}{l}\text { Circle, } \\
\text { Ellipse } \\
(6 \%) \\
\end{array}$ \\
\hline \multicolumn{4}{|c|}{ Perceptual Grouping of Variables } \\
\hline Prototypicality & High & Medium & Low \\
\hline
\end{tabular}




\begin{tabular}{|c|c|c|c|}
\hline Shape Choice & $\mid \begin{array}{l}\text { Rectangle } \\
(92 \%)\end{array}$ & - & \begin{tabular}{|l|} 
Ellipse, \\
circle, \\
pyramid \\
$(8 \%)$, curly \\
bracket, \\
arrow, \\
cloud
\end{tabular} \\
\hline $\begin{array}{r}\text { Shape } \\
\text { Demarcation } \\
\text { (Lines, Area } \\
\text { Color) }\end{array}$ & $\begin{array}{l}\text { Solid line } \\
(51 \%)\end{array}$ & $\begin{array}{l}\text { Dashed } \\
\text { line }(41 \%)\end{array}$ & $\begin{array}{l}\text { Miscellane } \\
\text { ous (e.g. } \\
\text { colored } \\
\text { area; } 8 \% \text { ), } \\
\text { without } \\
\text { demarcatio } \\
\text { n }\end{array}$ \\
\hline Labelling & \begin{tabular}{|l} 
Inside \\
common \\
region \\
$(75 \%)$ \\
\end{tabular} & \begin{tabular}{|l} 
Outside \\
common \\
region \\
$(19 \%)$ \\
\end{tabular} & $\begin{array}{l}\text { Directly on } \\
\text { common } \\
\text { region's } \\
\text { line }(6 \%) \\
\end{array}$ \\
\hline \multicolumn{4}{|c|}{$\begin{array}{l}\text { Relationship between Variables (Causal } \\
\text { Propositions) }\end{array}$} \\
\hline Prototypicality & High & Medium & Low \\
\hline Line Choice & $\begin{array}{l}\text { Solid line } \\
(83 \%)\end{array}$ & $\begin{array}{l}\text { Dashed } \\
\text { line }(15 \%)\end{array}$ & \begin{tabular}{|l} 
Double \\
solid line \\
$(2 \%)$
\end{tabular} \\
\hline $\begin{array}{r}\text { Connectedness } \\
\text { Other } \\
\text { Variables } \\
\text { (Independent, } \\
\text { Dependent, } \\
\text { Moderating, } \\
\text { Mediating) }\end{array}$ & $\begin{array}{l}\text { Connecte } \\
\text { d (97\%) }\end{array}$ & - & $\begin{array}{l}\text { Not } \\
\text { connected } \\
(3 \%)\end{array}$ \\
\hline $\begin{array}{r}\text { Connectedness } \\
\text { Control } \\
\text { Variable } \\
\end{array}$ & $\begin{array}{l}\text { Connecte } \\
\text { d }(66 \%)\end{array}$ & \begin{tabular}{|l|} 
Not \\
connected \\
$(34 \%)$
\end{tabular} & - \\
\hline $\begin{array}{r}\text { Relationship } \\
\text { Demarcation }\end{array}$ & $\begin{array}{l}\text { One type } \\
\text { of edge } \\
(74 \%)\end{array}$ & \begin{tabular}{|l|} 
Two types \\
of \\
different \\
edges \\
$(24 \%)$ \\
\end{tabular} & $\begin{array}{l}\text { Four types } \\
\text { of different } \\
\text { edges }(2 \%)\end{array}$ \\
\hline \multicolumn{4}{|c|}{ Occurrence of Labels for Hypotheses } \\
\hline Prototypicality & High & Medium & Low \\
\hline Labeling & $\begin{array}{l}\text { Abbreviat } \\
\text { ion with } \\
\text { numbers } \\
(47 \%)\end{array}$ & \begin{tabular}{|l|} 
Abbreviat \\
ion with \\
numbers \\
and letters \\
$(34 \%)$ \\
\end{tabular} & $\begin{array}{l}\text { No explicit } \\
\text { label } \\
(19 \%)\end{array}$ \\
\hline Direction & No $(65 \%)$ & \begin{tabular}{|l|} 
Yes (e.g. \\
plus/minu \\
s; $35 \%)$ \\
\end{tabular} & - \\
\hline \multicolumn{4}{|c|}{ Reading Direction } \\
\hline Prototypicality & High & Medium & Low \\
\hline Direction & $\begin{array}{l}\text { Left-to- } \\
\text { right } \\
(90 \%)\end{array}$ & - & $\begin{array}{l}\text { Top-to- } \\
\text { bottom } \\
(10 \%)\end{array}$ \\
\hline
\end{tabular}

\subsection{Limitations and Challenges}

The proposed approach to improving the comprehensibility of visual research models may have several limitations and face several challenges. First, relying on prototypicality for theoretical guidance in developing a unified model notation may result in the notation being optimized only for a particular demographic or time period, since what is prototypical changes over time and from one social group to another. Future research may need to repeat the analyses (Table 1) separately, e.g., for different sub-fields of IS.

Second, developing a unified model notation may be impractical for such a diverse research field as IS, e.g., because several prototypes (e.g. for SEM) - may exist: related research in web design showed that users view different webpage layouts as prototypical for different website categories [8]. However, our preliminary analysis suggests that only one visualnotation prototype likely exists for the IS research models. Future research will validate this observation. Another danger of a proposed notation could be that construct deficit (missing construct for an ontological concept) or symbol deficit (missing symbol for a semantic construct) could limit the possibilities of expression for researchers wishing to use it [15].

Third, we only analyzed a subset of visual properties of research models. We focused on those features in which we could observe variation in the data set. Future research may need to expand the subset to include, e.g., text (font types and sizes, and use of upper- and lowercase letters for different elements) and color properties. Adding them may further require enlarging the sample of models to analyze, possibly including the models from other fields using research models, such as psychology or management science, which would allow researchers to contrast the differences between these fields and IS.

Finally, prototypicality may need to be complemented as a guiding principle of the unified model notation with other design principles, such as higher perceptual discriminability or lower visual complexity (cf., the principles in [15]). Future research will establish the relative contribution of each principle to model comprehensibility, though prototypicality may also be crucial for adoption, as the resulting unified model notation could be readily adopted in the field without personnel re-training since it would be based on what the personnel was already used to. The different principles do not necessarily exclude or contradict each other, but rather complement each other: perceptual 
discrimination and visual complexity address the early, perceptual stages of mental processing, whereas prototypicality addresses the latter, cognitive stages

\section{Future Work}

Future work will consist of three steps. First, we will sample and analyze more visual research models to identify features of IS research model with high and low prototypicality. In an ontological analysis, we intend to identify relevant semantic constructs presented in the research models, and symbolic vocabulary used to represent them. We also will derive a meta-model for research models based on the identified feature space building upon the metamodel for causal theories by [4].

For the final dataset of research models to derive prototypical features, we intend to download articles from more volumes of the journals. Additionally, we plan to include more researchers when analyzing specific manifestations within defined feature spaces. This allows us to ensure that the results are independent of the observer and test for inter-rater reliability. At the moment, our research does not yet include the differentiation of whether SEM was applied or not. However, it may well be that other prototypical models exist for SEM or other statistical models since tools use visual modeling to specify the model. Accordingly, two types of prototypical research models may have to be developed, one created with and one without SEM.

Furthermore, we will also take a closer look at how interaction effects are visualized as mentioned by Mueller [4].

Second, we plan to conduct an online survey with a sample of consistent of three different target groups: novices, IS researchers and researchers from other disciplines. We want to include novices, e.g. information systems bachelor students who have had little contact with graphical research models yet, because they have therefore not yet been "brainwashed" to be familiar and prefer a common, but not intuitive representation of research models. It is also relevant from the point of view of the scientific exchange of different research disciplines to know how other research communities perceive prototypical research models of the IS discipline, this is why we include researchers from outside the discipline.

We plan to confront participants with pairwise comparisons of research models. In each pairwise comparison, one model is high on prototypicality, while the second model differs in that it has a non- prototypical feature with low prototypicality. To ease the visual comparison, we will highlight the visual difference between the two models, e.g., with color. We examine only one feature at a time (e.g., symbol choice for control variables) and keep all other elements constant to mitigate confounding variables. These paired comparisons will be based on the previously defined feature spaces. We will ask the participants to select the model they would prefer to read (or use) in a paper. Since prototypical features are faster to categorize [25], reaction times will be an indicator of the relevance of a specific feature for prototypicality. For each pairwise comparison, we will use a different research model. We intend to create three parallel versions of the experimental material, each using its own set of research models to exclude the possibility for a confounding effect of a particular research model.

Finally, we plan to assess comprehensibility of research models with low or high (visual) prototypical features in an eye-tracking experiment with a Tobii Spectrum using a between-subject design. Eye movements will also likely be descriptive of prototypicality, as less prototypical models could result in longer saccades, e.g., due to less-orderly information search patterns. Comprehension items will ask participants whether specific research hypotheses can be derived from a research model. Research models will be informationally equivalent to avoid confounding with the number of semantic constructs represented. In order to validate the content of our treatments, we have the participants rate the models with regard to three semantic differential parameters: exemplar goodness, typicality, and representativeness [20].

\section{Conclusion}

The paper proposed that from a theoretical perspective, prototypicality of research models should be advantageous in terms of comprehension and preference, especially for researchers within the IS discipline. We plan to test our developed hypotheses on the superiority of research models with prototypical features by conducting a survey and an eye-tracking experiment. In addition, our research will help to identify "constructive deviations", i.e. features of research models that are rarely used, but beneficial from a cognitive point of use to foster innovation and not just perpetuate the status quo. With the research proposed in this paper, we further intend to provide first insights on the use of graphical research models in the IS discipline, serving as a valuable initial contribution to opening the black box 
on which semantic constructs are typically represented and how they are visualized. This knowledge of current prototypical features of research models can serve as an empirical basis for developing a standardized graphical notation for research models in future work. Such a notation can improve research reporting and could also be of interest for other disciplines in the social sciences.

\section{References}

[1] J. Recker, Scientific research in information systems: a beginner's guide: Springer Science \& Business Media, 2012.

[2] B. Pajo, Introduction to research methods: a hands-on approach: SAGE Publications, 2017.

[3] G. Schraw and A. P. Gutierrez, "Examining the type, frequency, and interpretative complexity of visual displays appearing in the journal of educational psychology, 2010 2014," in Use of Visual Displays in Research and Testing: Coding, Interpreting, and Reporting Data, ed, 2015, p. 83.

[4] R. M. Mueller, "A Meta-model for Inferring Intertheory Relationships of Causal Theories," in 2015 48th Hawaii International Conference on System Sciences, 2015, pp. 4908-4917.

[5] H. H. Clark and S. E. Brennan, "Grounding in Communication," in Perspectives on Socially Shared Cognition, L. B. Resnick, et al., Eds., ed Hyattsville MD: American Psychological Association, 1991, pp. 127-149.

[6] R. Hirschheim, H. K. Klein and K. Lyytinen, Information Systems Development and Data Modeling: Conceptual and Philosophical Foundations. Cambridge, UK: University Press, 1995.

[7] L. Santa-Maria and M. C. Dyson, "The effect of violating visual conventions of a website on user performance and disorientation: how bad can it be?," in Proceedings of the 26th annual ACM international conference on Design of communication, 2008, pp. 47-54.

[8] S. P. Roth, et al., "Location matters, especially for nonsalient features-An eye-tracking study on the effects of web object placement on different types of websites," International journal of human-computer studies, vol. 71, pp. 228-235, 2013.

[9] K. Figl, "Comprehension of Procedural Visual Business Process Models," Business \& Information Systems Engineering, vol. 59, pp. 41-67, 2017.

[10] M. Scaife and Y. Rogers, "External cognition: how do graphical representations work?," Int. J. Hum.-Comput. Stud., vol. 45, pp. 185-213, 1996.

[11] R. Reber, N. Schwarz and P. Winkielman, "Processing fluency and aesthetic pleasure: Is beauty in the perceiver's processing experience?," Personality and Social Psychology Review, vol. 8, pp. 364-382, 2004.

[12] G. M. Spreitzer and S. Sonenshein, "Toward the construct definition of positive deviance," American behavioral scientist, vol. 47, pp. 828-847, 2004.

[13] A. Bhattacherjee, "Social science research: Principles, methods, and practices," 2012.
[14] L. Cao, B. Ramesh and M. Rossi, "Are DomainSpecific Models Easier to Maintain Than UML Models?," IEEE Software, vol. 26, pp. 19-21, 2009.

[15] D. L. Moody, "The "Physics" of Notations: Towards a Scientific Basis for Constructing Visual Notations in Software Engineering," IEEE Transactions on Software Engineering, vol. 35, pp. 756-779, 2009.

[16] S. Hitchman, "The details of conceptual modelling notations are important - a comparison of relationship normative language," Communications of the Association for Information Systems, vol. 9, 2002.

[17] R. M. Mueller, "Theory-Data Maps: A Meta-Model and Methods for Inferring and Visualizing Relationships between Causal Theories and Empirical Evidences," in 2016 49th Hawaii International Conference on System Sciences (HICSS), 2016, pp. 5288-5297.

[18] P. P.-S. Chen, "The entity-relationship model—toward a unified view of data," ACM Transactions on Database Systems (TODS), vol. 1, pp. 9-36, 1976.

[19] D. Moher, et al., "Preferred reporting items for systematic reviews and meta-analyses: the PRISMA statement," Annals of Internal Medicine, vol. 151, pp. 264269, 2009.

[20] B. Loken and J. Ward, "Alternative approaches to understanding the determinants of typicality," Journal of Consumer Research, vol. 17, pp. 111-126, 1990.

[21] E. Rosch and C. B. Mervis, "Family resemblances: Studies in the internal structure of categories," vol. 7, pp. 573-605, 1975.

[22] W. K. Hung and L. L. Chen, "Effects of novelty and its dimensions on aesthetic preference in product design," International Journal of Design, vol. 6, pp. 81-90, 2012.

[23] T. W. A. Whitfield, "Predicting preference for familiar, everyday objects: An experimental confrontation between two theories of aesthetic behaviour," Journal of Environmental Psychology, vol. 3, pp. 221-237, 1983.

[24] P. Hekkert, D. Snelders and P. C. Wieringen, "'Most advanced, yet acceptable': typicality and novelty as joint predictors of aesthetic preference in industrial design.," British Journal of Psychology, vol. 94, pp. 111-124, 2003.

[25] C. B. Mervis and E. Rosch, "Categorization of natural objects," Annual Review of Psychology, vol. 32, pp. 89115, 1981.

[26] K. Duncker and L. S. Lees, "On problem-solving," Psychological Monographs, vol. 58, 1945.

[27] D. G. Jansson and S. M. Smith, "Design fixation," Design Studies, vol. 12, pp. 3-11, 1991.

[28] D. K. Remler and G. G. Van Ryzin, Research methods in practice: Strategies for description and causation: Sage Publications, 2014.

[29] D. A. Pastor and S. J. Finney, "Promoting the use of path diagrams in quantitative research," in Use of Visual Displays in Research and Testing: Coding, Interpreting, and Reporting Data, ed, 2015, p. 111.

[30] R. Reber, "Processing fluency, aesthetic pleasure, and culturally shared taste," in Aesthetic Science: Connecting Minds, Brains, and Experience, ed Oxford: Oxford University Press, 2012, pp. 223-249.

[31] C. Martindale and K. Moore, "Priming, prototypicality, and preference," Journal of Experimental 
Psychology: Human Perception and Performance, vol. 14, pp. 661-670, 1988.

[32] P. Winkielman, et al., "Prototypes are attractive because they are easy on the mind," Psychological science, vol. 17, pp. 799-806, 2006.

[33] C. Ware, Information Visualization, 2 ed. San Francisco: Elsevier. Morgan Kaufmann, 2013.

[34] R. C. Anderson and P. D. Pearson, "A schematheoretic view of basic processes in reading comprehension," in Handbook of Reading Research, P. D. Pearson, et al., Eds., ed New York: Longman, 1984, pp. 255-291.

[35] M. B. McVee, K. Dunsmore and J. R. Gavelek, "Schema theory revisited," Review of Educational Research, vol. 75, pp. 531-566, 2005.

[36] S. E. Palmer, "Common region: A new principle of perceptual grouping," Cognitive Psychology, vol. 24, pp. 436-447, 1992 